\title{
Differences of Interest in Human Body Structures Among Paramedics from Various Specialized Fields: A Study Aiming at an Effective Anatomical Instruction
}

Noboru Sakamoto ( $\square$ mocchi427@yahoo.co.jp )

Chiba University https://orcid.org/0000-0002-5936-7371

Masatoshi Komiyama

Chiba University

Hidenobu Miyaso

Tokyo Medical University

\section{Yota Sugata}

Chiba University

Chisato Mori

Chiba University

Eiji Shimizu

Chiba University

Yoshiharu Matsuno

Intenational University of Health and Welfare

Research article

Keywords: anatomical education, anatomical practice, anatomical tour, human body structure, paramedical, interest

Posted Date: May 11th, 2020

DOl: https://doi.org/10.21203/rs.3.rs-27263/v1

License: (c) (i) This work is licensed under a Creative Commons Attribution 4.0 International License. Read Full License 


\section{Abstract}

This study aimed to identify human body areas in which paramedical students are particularly eager to observe and that are of interest in relation to the type of healthcare profession by means of a questionnaire after anatomical observation. A total of 840 students from 27 schools who visited Chiba University School of Medicine for anatomical tours, were examined after responding to the open questionnaire along with their consent. Terms of the human body structure that were of high interest were abstracted from the responses, and the frequency was analyzed according to individual specialized field. As a result, 1746 words were abstracted overall, and a high level of interest was identified in the nervous system (482 words), digestive system (425 words), cardiovascular system (305 words), musculoskeletal system ( 254 words), and respiratory system (183 words) sequentially. When considering individual specialized fields, the highest interest was found in the nervous system by nurses, acupuncturists, and emergency medical technicians, the digestive system by pharmacists and nutritionists, and the musculoskeletal system by rehabilitationists. When providing instruction during the observation, explanation should focus on areas of higher interest, as clarified in this study. At the same time, instruction in less interesting but are still important areas for the students in their specialized field must be provided with ingenuity to cultivate interest.

Mini-abstract: Highly interesting human body structures were the nervous system, digestive system, and musculoskeletal system, for nurses, acupuncturists, and emergency medical technicians; pharmacists and nutritionists; and the rehabilitation field, respectively.

\section{Introduction}

Understanding the structure of a human body is important for all healthcare professionals, whether they are doctors, dentists, or paramedics (Kobayashi 1998; Mc Garvey et al. 2015). In addition to anatomy lectures, anatomical practice is compulsory in medical and dental education in Japan. Medical and dental students can deepen their knowledge about the human body structure by performing numerous anatomical practices by themselves over a long period. Meanwhile, in paramedical education, only lectures on anatomy are compulsory, not anatomical practice. Considering these differences in the education system regarding anatomy, a significant gap has been observed in the level of understanding of the human anatomy between medical/dental students and paramedical students.

In addition to doctors and dentists, a wide range of professions, including nurses, physical therapists, occupational therapists, pharmacists, clinical technicians, acupuncturists, and moxibustion practitioners, has been covered by Japanese medical certification. As mentioned, unlike "anatomy lectures," which are compulsory, "anatomical practice" is not necessarily included in the curriculum at training schools for these healthcare professionals. However, recently, several schools have been actively incorporating programs that can further deepen the understanding of the human body structure. These programs include anatomical practices using human anatomical models and observational visits to medical and dental departments that perform anatomical tour on human cadavers. Although these programs can 
enhance the paramedical students' understanding of the human body, the educational effect might be limited because the anatomical models cannot provide the real-life shape and three-dimensional locations of the human organs. Meanwhile, during anatomical observation, they can see actual human organs. Thus, they can assess the real-life size and location of organs, feel the texture, membrane structure, and connective tissues, which cannot be exactly replicated in models, and gain knowledge about individual variations and changes due to aging or disease. In some reports published in Japan and other countries, anatomical observations can enhance the understanding of human anatomy (Kobayashi 1998; Doumoto et al. 1999; Azer and Eizenberg 2007; Anahara et al. 2008; Patel and Moxham 2008; Kimura et al. 2012; Sakamoto et al. 2016; Ghosh 2017b). Kawashiro et al. (2009) reported that anatomical observation is expected to increase the desire for employment and raise awareness among healthcare professionals. In addition, these anatomical observations can arouse a sense of awe regarding the human body and a sense of gratitude to the donors and their bereaved families (Kawashiro et al. 2009; Ghosh 2017a; Saw 2018). For these reasons, training schools for healthcare professionals (paramedical staff) in Japan actively incorporate anatomical observation in their curriculum (Doumoto et al. 1999; Kawamata et al. 2015) and implement observations with the aid of an anatomy laboratory at medical and dental departments.

The anatomy instructor of the medical or dental department, which provides the anatomical tours, or the escorting teacher of the training school for paramedical staff can deliver the lecture during anatomical observation. The anatomy instructor has a profound knowledge of the overall structure of the human body but does not necessarily understand which part of the human body is of particular interest to the visiting paramedical students, in relation to the specialized knowledge required in their respective profession. Meanwhile, the escorting teacher may be well versed in the area of interest of the students but not as familiar with the human body structure as the anatomy instructor. Furthermore, most training schools for paramedical staff provide approximately 2 hours long of anatomical observation for only a few times during the entire curriculum (Amimoto et al., 2017). Hence, to efficiently increase the educational effect of an anatomical observation for anatomical practice, the anatomy instructor, who is already familiar with the human body structure, should first understand the areas of interest of the visiting students. Through this initial step, the instructor can impart basic knowledge about body areas that the students are particularly interested and provide instruction on those areas in relation to the specialized knowledge of the students' profession. Regarding body areas in which the students may not be particularly interested, instruction must be provided in a way that arouses the interest of the students to gain the minimum required knowledge for their professions.

To realize the ideal instruction for observation, we need to first determine which body areas are of interest to the visiting paramedical students, and the relation of the areas to their profession. To date, the educational effects of anatomical observation for anatomy practices have been extensively reported, but only few reports describe the situation of anatomical observation by individual profession. Some reports targeted students from few professions, such as physical therapy and occupational therapy (Finn et al. 2018; Kinirons et al. 2018), but no reports have targeted a wide range of professions that track the relationship between profession and the area of interest. 
Therefore, this study used a questionnaire to determine which human body parts were of interest to students of training schools for paramedical staff. This study aimed to identify body areas that students are particularly eager to observe and that are of interest in relation to the type of their healthcare profession.

Currently, Japan has approximately 415,000 doctors and dentists, and 3,130,000 paramedics (Ministry of Health, Labor and Welfare, 2017). Deepening the knowledge on human body structure by paramedical staff, who are 7.5 times as many as doctors and dentists, before they interact with patients is especially important, considering that they will be directly involved in the treatment of patients with various disease types. The paramedical staff primarily handles the human body from the surface; for example, physical therapists treat muscles and joints, whereas nurses perform intravenous injection and blood-pressure measurement. However, to achieve effective treatment and care, these professionals must be able to thoroughly imagine the internal structure of the human body. This ability is crucial to prevent medical accidents.

\section{Materials And Methods}

\section{Subjects}

This study enrolled 878 paramedical students who participated in an anatomical observation practice at Chiba University from July 2013 to March 2016. The students were divided according to their study profession as follows: nurse training course, 519 students (426 vocational school students and 93 university students and graduate students; hereinafter referred to as "nurses"); acupuncture massage training course, 160 students ("acupuncturists"); pharmacist training course, 87 students ("pharmacists"); physical therapist/occupational therapist training course, 50 students ("rehabilitationists"); nutritionist training course, 46 college students ("nutritionists"); and emergency medical technician training, 16 students ("EMT") (Table 1). 


\begin{tabular}{|ll|}
\hline Specialized field & Number of persons \\
\hline Nurse & 519 \\
Vocational School (1st year) Student & $(266)$ \\
Vocational School (2nd year) Student & $(160)$ \\
University (2nd year) Student & $(65)$ \\
Graduate School Student & $(28)$ \\
\hline Acupuncturist & 160 \\
\hline Pharmacist & 87 \\
\hline Physical therapist, Occupational therapist & 50 \\
\hline Nutritionist & 46 \\
\hline Emergency medical technician training & 16 \\
\hline
\end{tabular}

Table 1

Specialized field and number of targeted students

\section{Anatomical observation training method}

Prior to starting the anatomical observation, students were briefed on the significance of body donation and ethics. Thereafter, they went to the anatomical practice room, were divided into 3 to 4 teams per group, and performed anatomical observation in a rotation method wherein the observers moved to the next station (session) every 15-20 minutes (Fig. 1). In session 1, heart, lungs, and major internal organs, such as large blood vessels, and the upper limbs of the study cadaver were observed using the practice body. In session 2, abdominal organs, such as the small and large intestines, pelvic organs, such as the uterus, and the muscles and nerves on the anterior aspect of the lower limbs were observed. In session 3 , the brain and spinal cord, which were removed, were observed. In session 4, the back (spinal cord and back muscles) and the muscles and nerves on the posterior aspect of the lower limbs were observed. In cases of a four-team system, sessions 1 to 4 were conducted for 15 minutes each, and in cases of a three-team system, sessions 1, 2, and $3+4$ were performed for 20 minutes each (Fig. 1). After observing all the sessions in rotation, we performed independent observation and Q\&A response for approximately 30 minutes. Although the cadavers used for observation varied yearly, the degree of dissection of the bodies remained the same.

\section{Questionnaire survey}

After observation, we conducted a questionnaire survey through an open questionnaire method. Regarding questionnaire survey contents, the students were allowed to freely describe the body areas 
which they had interest (which were considered instructive) within the range of observation. Moreover, we asked questions regarding their basic characteristics, such as their specialized field, college or vocational school, year, past observation experience, and clinical experience.

For the acquired responses, the contents of free description were classified according to human anatomy sections with similar meanings, and words indicating human body parts were extracted from the sentences in the free description contents. If words with identical meaning were described several times in the same context, they were counted as one.

The extracted words were first classified by type and then divided into small items. Furthermore, the smaller items were classified according to the organ system and then considered as large items.

\section{Ethical issues}

This study was approved by the Ethics Review Board (Chiba University, Graduate School of Medicine, Approval No. 1591; and Chiba University, Graduate School of Nursing, Approval No. 25-15). Subjects were informed in advance of the purpose and significance of the study, the voluntary nature of cooperation and that no disadvantage would occur when refusing to cooperate, protection of personal information, and more, both in written and verbal forms. Regarding cooperation, the student was asked to sign a consent form and to fill out and submit the questionnaire. In a case of impossible cooperation, the student was asked to return them in blank.

\section{Results}

\section{Overall results of the questionnaire}

The valid response rate of the questionnaire was $95.7 \%$ (number of respondents: 840 people). From the results of the questionnaire, 1746 words describing a human body part as the area of interest could be abstracted, amounting to 2.08 words in the free description per respondent.

Then, these 1746 words were categorized into 48 small items (Table 2). The most frequently used words in the small item categories included the circulatory organs ( 261 words), the brain ( 218 words), and the nervous system (160 words) (Fig. 2). Meanwhile, only one response each was received for adrenal glands and esophagus, and no responses were received for pancreas, oral cavity, nasal cavity, and sensory organs (Table 2). 


\begin{tabular}{|c|c|}
\hline Large items & Small items \\
\hline $\begin{array}{l}\text { 1. Nervous system } \\
(482)^{*}\end{array}$ & $\begin{array}{l}\text { 1. Brain (218) 2. Nervous system (160) 3. Spinal nerves (45) } \\
\text { (32) 5. Cranial nerves (27) }\end{array}$ \\
\hline
\end{tabular}

2. Digestive system $(418)$

6. Internal organs (101) 7. Small intestine (85) 8. Abdominal organs (64) 9. Digestive organs (61)

10. Large intestine (53) 11. Liver (39) 12. Stomach (14) 13. Esophagus (1)

3. Circulatory system (305)

14. Circulatory organs (261) 15 . Interior view of the heart (16) 16. Brain vessels (9) 17. Spleen (9)

18. Nutritional blood vessels (7) 19. Pericardium (2) 20. Exterior view of the heart (1)

4. Musculoskeletal system (254)

21. Musculoskeletal system (120) 22. Muscles of the lower limb (34) 23. Joints (26) 24. Muscles of the body trunk (16)

25. Muscles/bones of the upper limb (16) 26. Muscles/bones of the spine (16) 27. Ligaments (10)

28. Muscles/bones of the head and neck (8) 29. Spinal column (5) 30. Muscles/bones of the hip (3)

5. Respiratory system (183) 31. Lung (119) 32. Thoracic cavity (40) 33. Trachea/bronchi (19) 34. Respiratory organs (5)

6. Urogenital system (67)

35. Female organs (27) 36. Kidney (17) 37 . Male organs (7) 38 . Pelvic cavity (7) 39. Reproductive organs (4)

40. Bladder (3) 41. Urinary organs (2)

7. Endocrine system (5)

8. Other (32)

42. Pituitary gland (2) 43. Thyroid gland (2) 44. Adrenal gland (1)

45. Skin (18) 46. Membrane (7) 47. Fat (5) 48. Peritoneum (2)

*Figures in parentheses indicate the number of words included in the items.

Table 2

Areas of interest categorized into organ systems (large items) and types (small items)

The items could be divided into eight large items by categorizing small items into organ systems. Words that are most frequently mentioned were found in the following systems sequentially: the nervous system (482 words), digestive system (418 words), circulatory system (305 words), musculoskeletal system (254 words), respiratory system (183 words), urogenital system ( 67 words), endocrine system (5 words), and other (32 words). Items in "other" include the skin, membrane, fat, and peritoneum (Table 2).

\section{Questionnaire results by a specialized field}

Differences in areas of interest were observed in small and large items based on the specialized field. The top 3 items among the small items are shown below according to the specialized field (Fig. 3): 
All nurses (total responses, 1066 words): circulatory organs (193 words, $18.1 \%$ ), brain (138 words, 12.9\%), and nervous system (107 words, $10.0 \%)$.

Nurses by school year:

Vocational school student (1st year) (total responses, 557 words): circulatory organs (95 words, 17.1\%), brain (86 words, $15.4 \%$ ), nervous system (57 words, $10.2 \%$ )

Vocational school student (2nd year) (total responses, 315 words): circulatory organs (70 words, 22.2\%), brain (38 words, $12.1 \%$ ), nervous system (38 words, $12.1 \%$ )

University student (2nd year) (total responses, 129 words): circulatory organs (17 words, $13.2 \%)$, abdominal organs (17 words, $13.2 \%$ ), trachea and bronchus (15 words, $11.6 \%$ )

Graduate students (total responses, 65 words): circulatory organs (11 words, $16.9 \%$ ), abdominal organs (6 words, $9.2 \%$ ), digestive organs (5 words, $7.7 \%$ )

Acupuncturists (total responses, 335 words): nervous system (42 words, $12.5 \%$ ), musculoskeletal system (41 words, $12.2 \%)$, internal organs (31 words, 9.3\%)

Pharmacists (total responses, 181 words): circulatory organs (30 words, $16.6 \%$ ), brain ( 28 words, $15.5 \%$ ), lungs (24 words, $13.3 \%)$

Rehabilitationists (total responses, 80 words): internal organs (15 words, 18.8\%), musculoskeletal system (13 words, $16.3 \%$ ), muscles/bones of the lower limb (13 words, $16.3 \%$ )

Nutritionists (total responses, 61 words): circulatory organs (10 words, 16.4\%), brain (8 words, $13.1 \%)$, digestive organs (6 words, $9.8 \%$ )

EMT (total responses, 23 words): brain ( 6 words, $26.1 \%$ ), circulatory organs ( 5 words, $21.7 \%$ ), liver (4 words, $17.4 \%)$

The most frequent response received from all nurses for all year levels was the circulatory organs, followed by the brain and the nervous system, similar to vocational school students. In contrast, the order of frequency was abdominal organs, trachea, and bronchus for university students, and abdominal organs and digestive organs for graduate students. Additionally, as a characteristic of all small items, the brain was ranked within the top 3 in each specialized field, except for rehabilitationists.

As described above, the top 3 small items among the entire data were as follows sequentially: the circulatory organs, brain, and nervous system. A total of 261 students responded circulatory organs, of which 193 persons were nursing students; thus, nurses occupied a high percentage regarding these respondents. Consequently, the ratio of respondents for circulatory organs, brain, and nervous system, which occupied a high percentage in small items, within the total respondents was calculated according to individual specialized field (Fig. 4). Aside from nurses (193/519 respondents, 37.2\%), many of the 
pharmacists (30/87 respondents, $34.5 \%)$ and EMT (5/16 respondents, $31.3 \%)$ responded "circulatory organs." Regarding the brain, EMT (6/16 respondents, $37.5 \%)$ and pharmacists (28/87 respondents, $32.2 \%)$ had high response rates in contrast with many other specialized fields, including nurses $(138 / 519$ respondents, $26.6 \%$ ) and nutritionists (8/46 respondents, $17.4 \%)$. With regard to the nervous system, compared with nurses (107/519 respondents, $20.6 \%$ ) and acupuncturists (42/160 respondents, $26.3 \%)$, rehabilitationists responded at $10.0 \%$ (5/50 respondents), pharmacists (4/87 respondents) and nutritionists (2/46 respondents) responded at less than 5.0\%, and EMT responded none (Fig. 4).

Next, the top 3 large items are shown below according to specialized field:

Nurses (total responses, 1066 words): nervous system (307 words, 28.8\%), digestive system (268 words, $25.1 \%)$, circulatory system (214 words, $20.1 \%)$

Acupuncturists (total responses, 335 words): nervous system (107 words, 31.9\%), musculoskeletal system (102 words, $30.4 \%$ ), digestive system (55 words, $16.4 \%$ )

Pharmacists (total responses, 181 words): digestive system (66 words, 36.5\%), circulatory system (36 words, $19.9 \%$ ), nervous system (34 words, $18.8 \%$ )

Rehabilitationists (total responses, 80 words): musculoskeletal system (42 words, $52.5 \%$ ), digestive system (18 words, $22.5 \%)$, nervous system (15 words, $18.8 \%$ )

Nutritionists (total responses, 61 words): digestive system (21 words, $34.4 \%)$, circulatory system (12 words, $19.7 \%$ ), nervous system (10 words, $16.4 \%$ )

EMT (total responses, 23 words): nervous system (9 words, 39.1\%), circulatory system (7 words, 30.4\%), digestive system ( 6 words, $26.1 \%$ )

The most frequent response was the nervous system by nurses, acupuncturists, and EMT; the digestive system by nutritionists; and the musculoskeletal system by rehabilitationists.

The ratio of respondents for large items was also analyzed in each specialized field (Fig. 5). Although nurses (307/519 respondents, $59.2 \%)$, acupuncturists (107/160 respondents, $66.9 \%)$, and EMT (9/16 respondents, $56.3 \%$ ) had high response rates for the nervous system, other fields had low response rates. For the digestive system, the response rate of pharmacists was $75.9 \%$, whereas those of other fields remained at $34.4 \%-48.6 \%$. Figures for the circulatory system ranged from $2.0 \%-43.8 \%$, where nurses, pharmacists, and EMT had relatively high response rates. Rehabilitationists, however, had an extremely low rate $(2.0 \%)$. Regarding the musculoskeletal system, acupuncturists and rehabilitationists had rates of $64.3 \%$ and $84.0 \%$, respectively, but other fields exhibited rates less than $20 \%$. For the respiratory system, pharmacists had the highest rate (39.1\%), followed by nurses $(22.7 \%)$; meanwhile, other fields showed rates less than $20 \%$.

\section{Discussion}


This questionnaire survey on areas of interest was given to paramedical students who participated in an anatomical observation at Chiba University. Differences between specialized fields of the students were considered according to the survey results.

Among the 48 summarized small items, the largest number of responses was obtained by the circulatory organs, followed by the brain and the nervous system, accounting for $36.6 \%$ of all responses. If the lung (the fifth largest number of responses) is included, the responses accounted for $43.4 \%$ of all responses. Paramedical students seem to be highly interested in organs essential to life. Meanwhile, only one response was received for the adrenal glands and esophagus, and no responses were received for the oral cavity, nasal cavity, and pancreas. Regarding oral and nasal cavities, no responses were received, considering that these body areas were not dissected and were excluded in the observation sessions.

Among the large items, the largest number of responses was obtained by the nervous system, followed by the digestive system, circulatory system, musculoskeletal system, respiratory system, urogenital system, endocrine system, and others. Interestingly, not all related organs are included in the large items. For instance, although the digestive system is a large category and has the second largest number of responses, very few students considered the observation of digestive organs, such as the esophagus, as instructive (highly interesting). The reason may be that the esophagus did not leave a deep impression because it could only be partially viewed, lying behind the trachea and pericardium. Regarding respiratory organs, students were less interested on the oral cavity and nasal cavity, probably because such organs had not been dissected and explained even during lecture times. Nonetheless, students had been taught about the structure and function of the trachea, bronchi, and lungs.

Moreover, analysis results revealed that students were interested in specific areas depending on their specialized field.

Among the small items, the circulatory organs obtained the highest interest according to nurses. Nursing students practice intravenous injections with their fellow students during practical exercises at schools but reportedly find such practice difficult because of indecision in choosing the right vein and of anxiety and nervousness regarding paracentesis (Kaminari et al. 2012). Furthermore, in the nursing field, knowledge on palpation, tapping methods for injection, cardiac and lung auscultation, and other circulatory organ-related information is indispensable, and linking human anatomy to clinical evaluation is important (Johnston 2010). Therefore, chances to learn about the circulatory organs at lectures and in practical exercises promote students to be interested on the circulatory organs. In other words, the circulatory organs, including veins of the cadavers, seemed to attract great interest during anatomical observation because the students have few chances to directly observe actual organs, even though the importance of anatomical knowledge of the circulatory system, including the course of veins, is stressed in classes and the actual clinical setting.

Among the large items, the nervous system obtained the highest interest in nurses. Within this category, the brain acquired many responses. The brain was a frequent response not only from nurses but also from all students in other specialized fields (Fig. 4). In this observation training, students can handle a 
real formalin-fixed brain sample and acquire detailed observation. Although none of the specialized fields directly work with the brain, the brain is the central organ for thought and behavior, and the appearance and actual handling of the brain had a strong impact on its importance and the experience of the students.

According to nursing students in a previous study, their understanding of the human anatomy was deepened by viewing a dissected body, the structure of the mediastinum, the size of the heart and structure of the pericardium, the state of the diaphragm, the position of the pelvic organs, such as the uterus, the relationship of the duodenum and pancreas, and more (Doumoto et al. 1999). In the current study, the circulatory system, brain, and nervous system were mentioned as the primary areas of interest, relatively different from those in the previous study mentioned above. This dissimilarity may reflect the difference in areas that could be observed and lectured. Given that the school year was not mentioned in the previous research, students of whichever year were interested in areas that could not be ascertained. First- and second-year vocational school students, second-year university students, and graduate students participated as nursing students (Table 1). The areas of interest between vocational college students, university students, and graduate students were different. Vocational school students and university students increasingly receive lectures as their grade increases; thus, differences in knowledge inevitably appear. Graduate students already have clinical experiences; hence, the areas of interest may vary as well. However, the circulatory organ ranked the highest in terms of areas of interest by participants in all year levels, possibly owing to the many opportunities that consider circulatory organs in lectures and practical exercises. These results should be reflected to future instruction methods, such as changing the teaching contents of anatomy according to each year, in the nursing field.

Meanwhile, many responses were received from acupuncturists for small items regarding the nervous system, musculoskeletal system, and internal organs consecutively. According to a previous literature, their interest was high according to depth, from the body surface to the internal organs, and the actual attachment structure of muscles to joints, and especially, in areas commonly associated with acupuncture and moxibustion, and areas where accidents easily occur during acupuncture (Suzuki et al. 2010). In relation to the results of the present study, the word "nervous system" can be used as the answer in consideration of the risk of accidental acupuncture to the nerve, and "internal organs" as the consideration of acupuncture depth, that is, student may be considering the aspect of the distance from the body surface to the internal organs when performing acupuncture. Thus, acupuncturists seemed to have been participating in the observation while imagining clinical practice.

Meanwhile, pharmacist students frequently responded about the digestive system, nervous system, and respiratory system. Notably, only the pharmacists included the respiratory system as one of the top 3 items of areas of interest. Pharmacists are expected to contribute to the proper use of drugs and early detection of side effects as "drug experts." Hence, physical assessment is required; for example, they should be able to detect respiratory side effects, such as drug-induced lung injury, at an early stage (Sakai 2015). 
With regard to rehabilitationists, many responses were received for small items regarding internal organs, musculoskeletal system, and muscles/bones of the lower limbs consecutively. In previous studies, physical/occupational therapy students demonstrated a higher interest in muscles, such as the origin and insertion point of the anterior serratus muscle, superficial and deep digital flexor tendons, thoracolumbar fascia, and the iliotibial tract (Doumoto et al. 1999; Schofield 2014; Schofield 2018). In the current study, internal organs ranked the highest, but the musculoskeletal system had almost the same level of interest as internal organs, similar to the results of previous reports. Regarding specialized rehabilitation work, such as a physical therapist, anatomy is an essential basic subject (Tozaki et al. 1997; Otani 1998). In addition, the musculoskeletal system is related to many subject areas, such as the range-of-motion test, manual muscle test, and kinesiology, in the rehabilitation work, suggesting the reason for the rehabilitationists' strong interest in the musculoskeletal system.

Moreover, nutritionists frequently responded about large items, namely, the gastrointestinal system, cardiovascular system, and nervous system. In the training course for nutritionists, anatomy and physiology are affiliated with the field "Human body structure, function, and disease composition" (Okada 2011). Methods using animals, such as mice and pigs, and visiting to a museum are mainly used for anatomical training, while opportunities for human body anatomical observation are only few (Shimizu et al. 2002; Okada 2011). In previous studies, students were strongly interested in understanding bone structure and breathing during practice using animals; however, such interest was not in proportion to interest in body areas that they personally considered needed (Okada 2011). In the same study, the areas of interest during a human body anatomical practice were not described, but the areas of interest for the human body and for animals were found to have a difference. When museum visiting and human body anatomical practice were compared, a stronger impact was reportedly felt by students during human body anatomical observation (Shimizu et al. 2002). Using the human body as the observation subject help students develop a deeper understanding of the internal structure compared with using animals.

In EMT, many responses were received regarding the nervous system, cardiovascular system, and gastrointestinal system consecutively. Currently in Japan, the range of emergency lifesaving procedures, which can be performed by EMT, is regulated by the Ministry of Health, Labor and Welfare, and it includes securing an intravenous line, securing the airway by intubation with an endotracheal tube, and administering epinephrine. When examining the relationship between these specific actions and the areas of interest, the cardiovascular system may be connected but none on other items. In addition, considering that the sample number of EMT in this study was low at 16 persons, the abovementioned connection may not be credible. Thus, a higher sample size is needed for analysis.

To date, the areas of interest have been considered according to individual small items, large items, and to individual specialized fields. Responses that obtained a higher rank in each individual field reflect the characteristic lectures and practice training of each field. The fact that responses show bias toward each specialized field must be an important information when considering an appropriate instruction customized for each specialized field, and to thoroughly provide guidance in future training instructions. 
Areas with relatively low interest included the adrenal glands, esophagus, oral or nasal cavity, spleen, and sensory organs, possibly because these areas were difficult to view (adrenal gland, esophagus, and spleen) or were not being focused during observation (oral, nasal cavity, spleen, and sensory organs). Presuming that the students of each specialized field will work at a clinical site in the future, knowledge on organs such as adrenal glands, esophagus, and spleen is important for nutritionists and pharmacists, apart from the digestive function and hormonal secretion. For rehabilitation professions, such as physical therapists, the oral and nasal cavities are important for respiratory function evaluation and treatment. Thus, identification of areas with low interest is also important for the possibility of changing the training instruction method to cover such deficiency. Moreover, considering that interest is biased according to individual specialized field, knowledge on a specific region can be possibly deepened by thorough explanation and spending more time on such areas of interest during overall explanation.

As mentioned at the beginning, instructors of a medical or dental department have a profound knowledge on the overall human body structure but may not fully grasp what areas are of particular interest to the visiting paramedical students in relation to the specialized knowledge required in their respective profession. Meanwhile, the escorting teachers of a training school may be well versed in the area of interest of the students but not as familiar with the human body structure as the anatomy instructor. In response to this situation, various trials on anatomical observation training method have begun in Japan and abroad (Ide 2004; Manyama et al. 2016; Alvarez and Schultz 2018; Rhodes et al. 2018; Smith et al. 2018).

In this study, areas in which students had interest and those in which students did not have interest have been identified according to individual specialized field. In anatomical observation practice, having interest in all body organs would be ideal, but it would also have limitations. One is the time constraint for practice; hence, the time allotted for observation is also limited. Given that observation training uses time when anatomical practice for medical students is not performed, frequency cannot be ensured. For this study, questionnaire survey and analysis were conducted under such restriction, and one limitation to this study is that areas of interest are within "the range of what can be seen." Moreover, given the varied sample number of each field, the sample size must be increased for a more accurate analysis. According to Matsuno et al. (2002), approximately $90 \%$ of the students who participated in anatomical observation responded that such practice is necessary. However, students who understood the lecture and stayed were approximately $70 \%$. Improvement in lecture appeal and the educational material is also important. Thus, efforts providing more effective anatomical observation instruction in the future, in a way that deepens the understanding of the students, are desired.

\section{Declaration}

\section{Acknowledgments}

We would like to thank the paramedical students who participated in this study. We also wish to express our thanks to the members of the Chiba Shiragiku community who donated their bodies to science. 


\section{Conflict of Interest:}

The authors declare that they have no conflict of interest.

\section{References}

1. Alvarez S, Schultz JH (2018) Professional and personal competency development in near-peer tutors of gross anatomy: A longitudinal mixed-methods study. Anat Sci Educ 11:282-293

2. Amimoto K, Uematsu M, Kawakami K, Hidaka M, Arakawa T. 2017. Report of a questionnaire on human anatomy practice. Japanese Physical Therapy Association.

URL:http://www.japanpt.or.jp/upload/japanpt/ obj/files/chosa/zintaikabo_Q_2017.pdf [accessed 10 October 2019] (In Japanese. Title was translated by the authors of the present study).

3. Anahara R, Kawashiro Y, Matsuno Y, Mori C, Kohno T (2008) The physical therapy undergraduate students' responses to the gross human anatomy subjects. Kaibogaku Zasshi 83:81-86

4. Azer SA, Eizenberg N (2007) Do we need dissection in an integrated problem-based learning medical course? Perceptions of first- and second-year students. Surg Radiol Anat 29:173-80

5. Doumoto T, Kawamada S, Yasuda M (1999) What do medical technician students learn from human body anatomical observation? - From descriptions on their impression, by students at three faculties of Hiroshima Prefectural College of Health and Welfare -. Kaibogaku Zasshi 72:643-647 (In Japanese. Title was translated by the authors of the present study)

6. Finn GM, Connolly SA, Gillingwater TH, Smith CF (2018) Putting gross anatomy into the curriculum: New anatomy syllabi for nursing and pharmacy students. Anat Sci Educ 11:427-428

7. Ghosh SK (2017a) Cadaveric dissection as an educational tool for anatomical sciences in the 21st century. Anat Sci Educ 10:286-299

8. Ghosh SK (2017b) Paying respect to human cadavers: We owe this to the first teacher in anatomy. Ann Anat 211: 129-134

9. Johnston AN (2010) Anatomy for nurses: providing students with the best learning experience. Nurse Educ Pract 10:222-226

10. Kaminari Y, Ichijo A, Masuda $Y$ (2012) Education methods for nursing students when performing their first venous blood specimen collection. Asahikawa Medical College Research Forum 13:104-111 (In Japanese. Title was translated by the authors of the present study)

11. Kawamata S, Aoysms H, Kurose T (2015) Importance of a single observation of dissected cadavers for nursing students. Structure and Function 14:12-21

12. Kawashiro Y, Anahara R, Kohno T, Mori C, Matsuno Y (2009) Attitudes of healthcare students on gross anatomy laboratory sessions. Anat Sci Educ 2:273-279

13. Kimura T, Matsuda W, Aimi Y, Taki K, Honma S, Udagawa J, Kudo K (2012) Visit to the human anatomical dissection course is effective for PT/OT student: Efficacy and evaluation. Structure and Function 11:24-32 
14. Kinirons SA, Reddin VM, Maguffin J (2018) Effects of alternating dissection with peer teaching and faculty prosected cadaver demonstrations in a physical therapy and occupational therapy gross anatomy course. Anat Sci Educ 0:1-10

15. Kobayashi K (1998) Importance of human dissection in the education of co-medical professionals and proposal for the improvement-expecting new era. Kaibogaku Zasshi 73:275-280

16. Manyama M, Stafford R, Mazyala E, Mazyala E, Lukanima A, Magele N, Kidenya BR, Kimwaga E, Msuya S, Kauki J (2016) Improving gross anatomy learning using reciprocal peer teaching. BMC Med Educ 16:95

17. Matsuno Y, Komiyama M, Kadota T, Kawabata Y, Ono Y, Sato K, Adachi T, Mori C (2002) Survey on the attitudes of co-medical students at Chiba University toward observation of dissection of human bodies Kaibogaku Zasshi 77:77-80

18. Mc Garvey A, Hicky A, Conroy R (2015) The anatomy room: a positive learning experience for nursing students. Nurse Educ Today 35:245-250

19. Ministry of Hearth, Labour and Welfare (2017) Annual Health, Labour and Welfare Report 2017. P45. URL:https://www.mhlw.go.jp/english/wp/wp-hw11/dl/02e.pdf [accessed 18 October 2018]

20. Otani $O$ (1998) Anatomy education for co-medical students - From the viewpoint of the anatomy department in medical school. Kaibogaku Zasshi 73:293-297 (In Japanese)

21. Okada M (2011) A study on devising effective laboratory courses in anatomy and physiology to train dietitians. Hiroshima Bunka Gakuen kiyo 43:33-42 (In Japanese)

22. Patel KM, Moxham BJ (2008). The relationships between learning outcomes and methods of teaching anatomy as perceived by professional anatomists. Clin Anat 21:182-189

23. Rhodes D, Fogg QA, Lazarus MD (2018) Dissecting the role of sessional anatomy teachers: A systematic literature review. Anat Sci Educ 11:410-426

24. Sakamoto H, Kawate T, Shimo S, Sekiguchi Y, SEI S (2016) A study on the needs of practical training for healthcare professionals, using anatomical specimens of human bodies in 2015 . Health Science University kiyo 12:25-36 (In Japanese)

25. Sakai $H$ (2015) Basic techniques for pharmacists in order to properly use drugs and medicines. Introduction and development of physical assessment -. Farumashia 51:835-840 (In Japanese. Title was translated by the authors of the present study)

26. Saw A (2018) A new approach to body donation for medical education: The silent mentor programme. Malays Orthop J 12:68-72

27. Schofield KA (2014) Anatomy in occupational therapy program curriculum: practitioners' perspectives. Anat Sci Educ 7:97-106

28. Schofield KA. (2018) Anatomy education in occupational therapy curricula: Perspectives of practitioners in the United States. Anat Sci Educ 11:243-253

29. Shimizu M, Fujii Y, Katabami Y (2002) Significance of visit of prospective dietitians at a biomedical museum -A comparison with the participation in dissection of human bodies. The Bulletin of the 
Faculty of Human Life Sciences, Fuji Women's University No.40:13-22 (In Japanese)

30. Smith CF, Tollemache N, Covill D, Johnston M (2018) Take away body parts! An investigation into the use of 3D-printed anatomical models in undergraduate anatomy education. Anat Sci Educ 11:44-53

31. Suzuki M, Sugata Y, Yakame S, Saito H, Sakamoto A (2010) The need for anatomical observation by practitioners of acupuncture and moxibustion - from standpoint of teachers of acupuncture and moxibustion schools. The program \& abstracts of the 59th Annual Meeting of the Japan Society of Acupuncture and Moxibustion, June 11-13 Osaka in Japan. (In Japanese. Title was translated by the authors of the present study)

32. Tozaki A, Kobayashi K, Shioda T, Takagi H, Watanabe H (1997) Survey of actual conditions of human body related education (anatomy) in medical technician training agencies. Kaibogaku Zasshi 72: 477 (In Japanese. Title was translated by the authors of the present study)

\section{Figures}



\section{Figure 1}

Rotation style autopsy observation method. 




Figure 2

Top 10 small items (out of 48 types) according to the number of included words.

(\%)

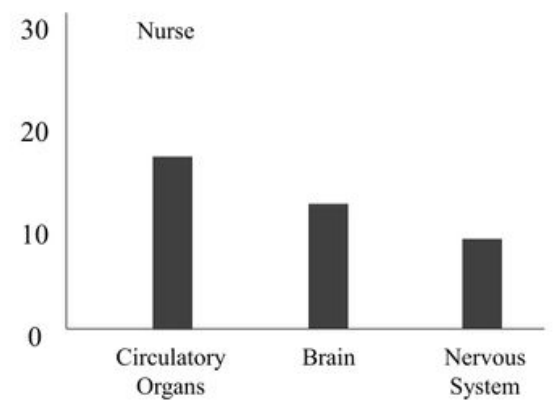

(\%)

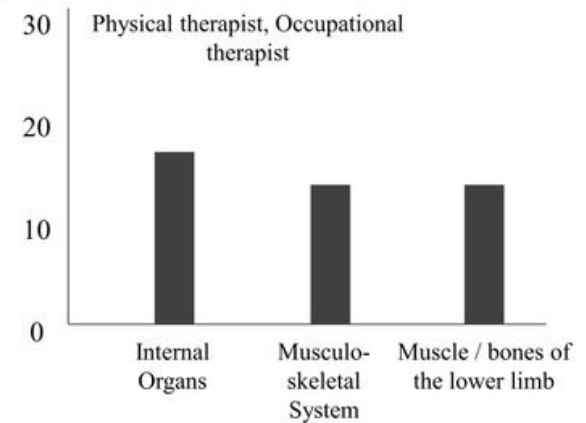

(\%)

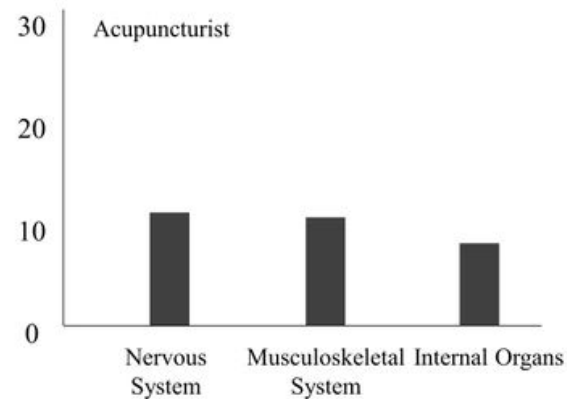

(\%)

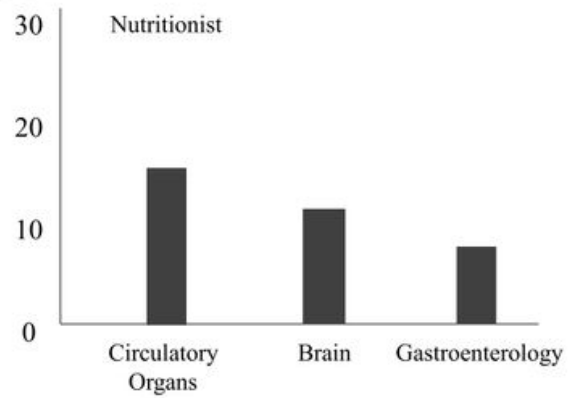

(\%)

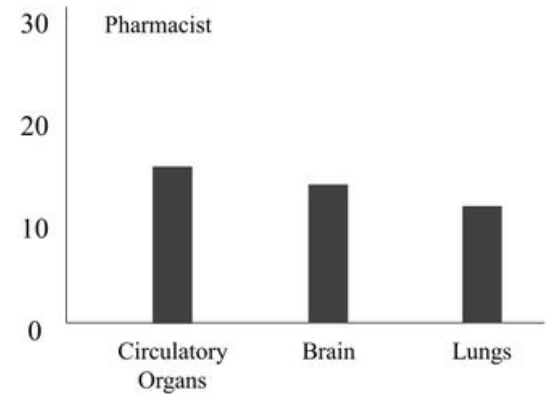

(\%)



Figure 3 
Top 3 small items in each specialized field according to the number of words. Percentage indicates the ratio of the number of words included in the small item against the total number of responded words in each specialized field. EMT, emergency medical technician training.

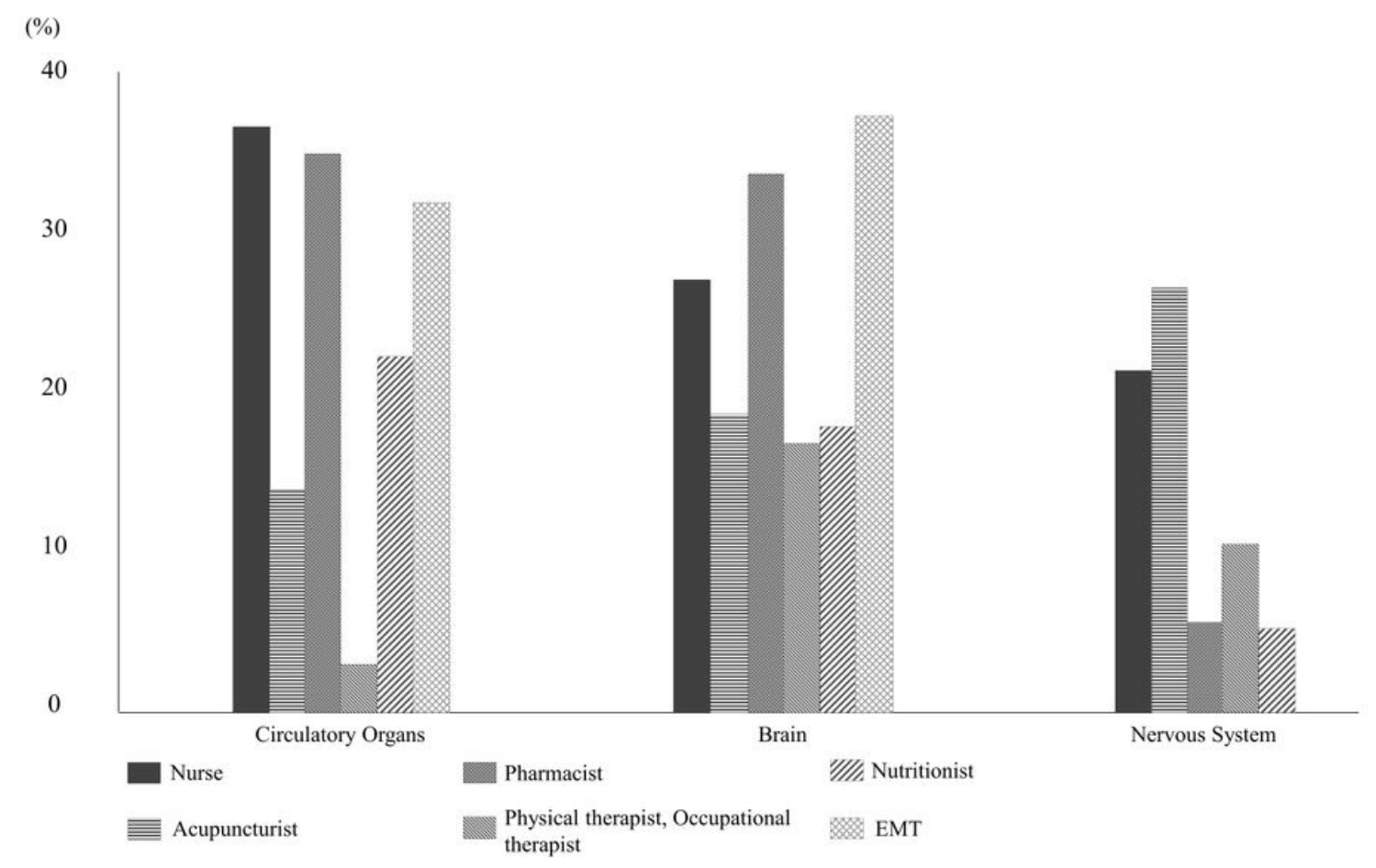

Figure 4

Comparison of respondent ratio among specialized fields in the top 3 small items among the entire data. Percentage indicates the ratio of the number of respondents included in the small item against the total number of respondents in each specialized field. EMT, emergency medical technician training. 


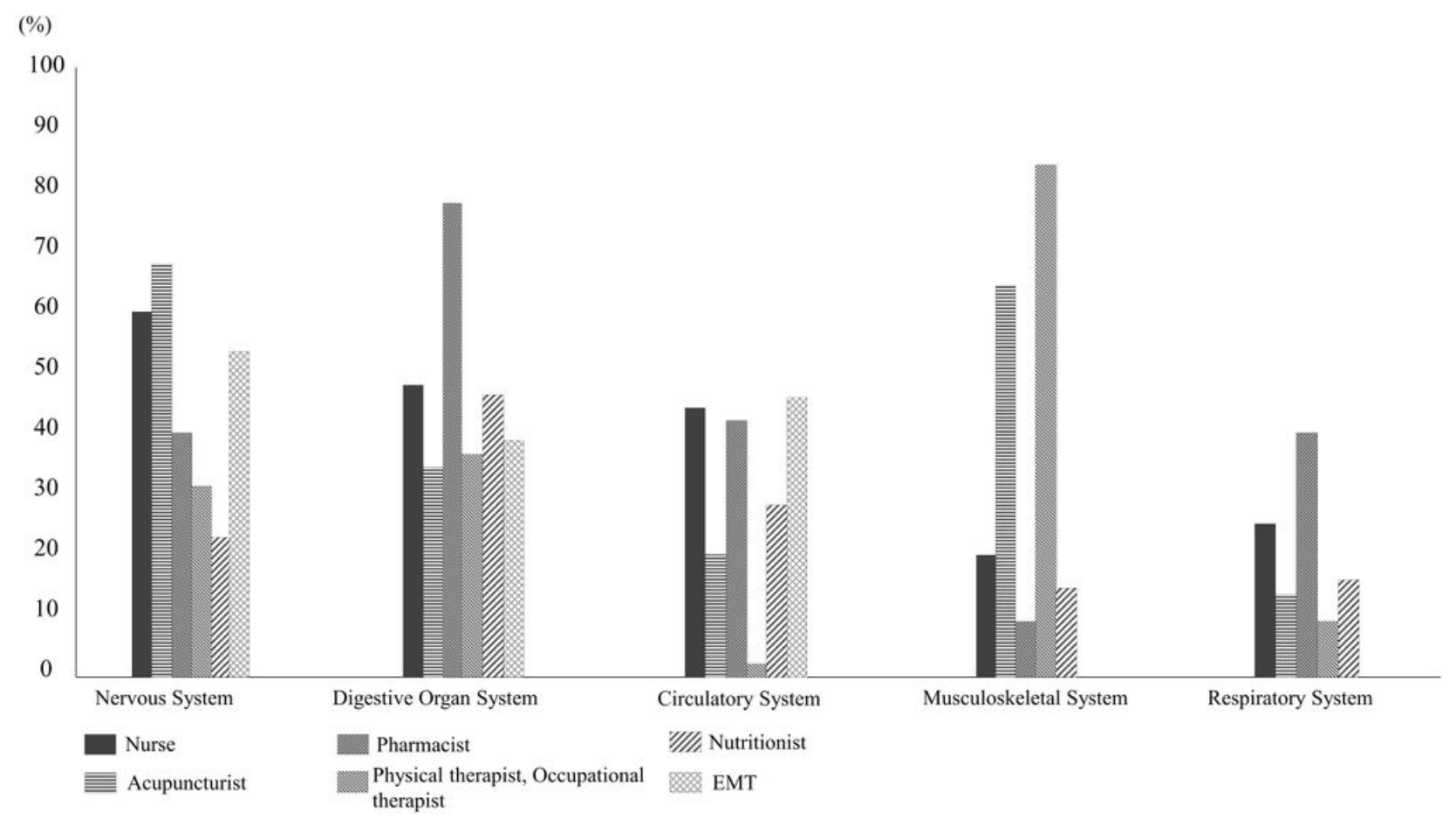

\section{Figure 5}

Comparison of respondent ratio among specialized fields in the top 5 large items. Percentage indicates the ratio of the number of respondents included in the large item against the total number of respondents in each specialized field. EMT, emergency medical technician training.

\section{Supplementary Files}

This is a list of supplementary files associated with this preprint. Click to download.

- CertificationForm.pdf 